\title{
Evidence for the AGN nature of LINERs
}

\author{
O. González-Martín, ${ }^{1}$ J. Masegosa, ${ }^{1}$ I. Márquez ${ }^{1}$ \\ and E. Jiménez-Bailón ${ }^{2}$ \\ ${ }^{1}$ Instituto de Astrofísica de Andalucía (CSIC), Apdo 3004, 10080 Granada, Spain \\ ${ }^{2}$ Università degli Studi Roma Tre, Rome, Italy \\ email: omaira@iaa.es
}

\begin{abstract}
We report the analysis of the X-ray data for a sample of 48 LINER nuclei with available X-ray Chandra imaging. In González-Martín et al. 200621 objects had enough count rate to make the spectral analysis. Here we enlarge the sample performing the spectral analysis of the XMM-Newton observations of 7 additional galaxies. Our aim is to investigate the physical mechanisms which power the nuclear activity of LINERs. The use of multiwavelength information at radio, UV, optical HST and X-ray lead us to conclude that at least $60 \%$ of the LINERs are hosting a low luminosity AGN in their nuclei.
\end{abstract}

Keywords. Galaxies: active - X-rays: galaxies

\section{Introduction}

LINERs are very common in the nearby universe. Pioneering works already estimated that at least $1 / 3$ of all the spiral galaxies are LINERs (Heckman et al. 1980). Nowadays, there is still an ongoing strong debate on the origin of the energy source in LINERs, with two main alternatives for the ionizing source being explored: either it is a low luminosity AGN, or it has a thermal origin related to massive star formation and/or from shock heating mechanisms resulting from the massive stars evolution. The search for a compact X-ray nucleus in LINERs is indeed one of the most convincing evidence about their AGN nature.

\section{X-ray analysis}

The sample has been grouped into two categories, attending to the X-ray morphology: (i) AGN-like Nuclei: unresolved point-like source in 4.5-8.0 keV band; (ii) Starburst-like Nuclei: without unresolved point-like source in 4.5-8.0 keV band. Two models (thermal and non-thermal), and their combination, were tested to account for the spectral emission of the objects with enough S/N (21 from Chandra, see González-Martín et al. 2006, and 7 from XMM-Newton). A combination of thermal and non-thermal components is required in most of the cases. The resulting median spectral parameters are $k T=0.7 \pm 0.2 \mathrm{keV}$ and $\Gamma=1.7 \pm 0.4$. Nuclear luminosities were either calculated from the best-fit model or estimated otherwise (assuming a power law of $\Gamma=1.8$ and galactic absorption). Fig. 1 (left) shows that AGN-like nuclei tend to be more luminous than SB-like nuclei.

When the fitting was not possible (20 objects) color-color (C-C) diagrams were used to estimate $\Gamma, k T$, and $n_{\mathrm{H}}$. Fig 1 shows the comparison between estimated and fitted values. Excepting three cases, the $\mathrm{C}-\mathrm{C}$ diagrams provide a good $\mathrm{T}$ estimation, whereas $\Gamma$ results to be slightly underestimated. The same result is obtained when XMM-Newton data are included. 

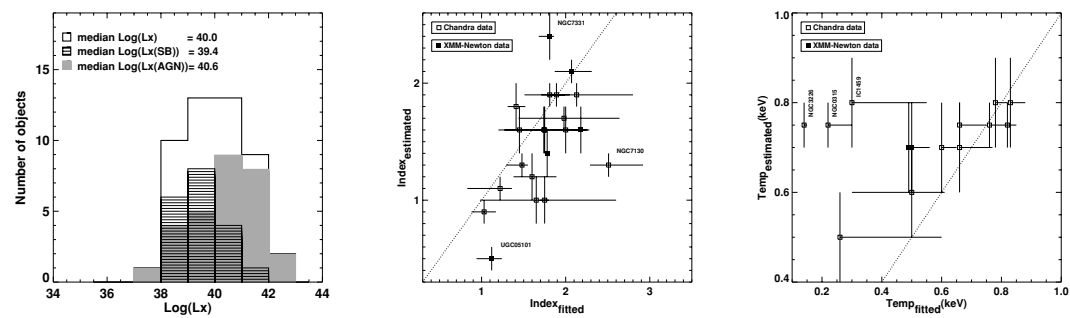

Figure 1. Left: Luminosity histogram for the whole sample (empty); objects classified as AGN-like (grey) and Starburst-like objects (dashed). Comparison between power index (centre) and temperature (right) as estimated from $\mathrm{C}-\mathrm{C}$ diagrams with those from fitted values.

\section{Other evidence}

In order to gain insight into the emission mechanisms in these objects, we looked for other evidence of the AGN nature in other wavelengths:

HST analysis. By Sharp-divided imaging the objects are classified into two groups: (a) compact nuclear sources (35 objects); and (b) dusty nuclear regions (8). All the galaxies classified as AGN by the X-ray imaging analysis show compact nuclei at the resolution of HST images.

Radio evidence. An unresolved nuclear radio core and flat continuum have been suggested as an evidence of AGN nature. Most of the LINERs in our sample classified by Filho et al. (2000) as AGNs in radio (13 objects) form an AGN-like class in X-rays (9).

$U V$ variability. For the 7 objects in common with the sample of 17 LINER galaxies with HST/UV data by Maoz et al. (2005), five show time variability hinting to their AGN nature; all of them belong to our AGN-like class.

Stellar populations. High Mass X-ray Binaries are not expected to be an important ingredient for the nuclear X-ray emission. For the 14 galaxies with data available (CidFernandes et al. 2004) the contribution of young stars is always less than $3 \%$.

Compton thickness. $L_{X} / L[\mathrm{OIII}]<1$ may be used to detect Compton-thick sources. In our sample, SB-like nuclei show lower values than AGN-like (mean value of 0.96 for SB candidates and 11.0 for AGN candidates). The high percentage of SB-like in the region occupied by Compton-thick objects (50\%) imply that they may host a strongly obscured AGN.

\section{Conclusions}

Although contributions from HMXBs and ULXs cannot be ruled out for some galaxies, we concluded in González-Martín et al. (2006) that $60 \%$ seems to be a lower limit for LINERs hosting an AGNs. The analysis of the Compton Thickness in our enlarged sample hints to a high percentage of our SB-like hosting strongly obscured AGNs.

This work was financed by DGICyT grants AYA2003-00128 and the Junta de Andalucía TIC114. OGM acknowledges financial support by the Ministerio de Educación y Ciencia through the Spanish grant FPI BES-2004-5044.

\section{References}

Cid Fernandes, R., Gonzalez Delgado, R. M., Schmitt, H. et al. 2004, ApJ, 605, 105

Filho, M. E., Barthel, P. D. \& Ho, L. C. 2000, ApJS, 129, 93

González-Martín, O. et al. 2006, A\&A in press (astro-ph/0605629)

Heckman, T. M., Crane, P. C. \& Balick, B. 1980, A\&A, 40, 295

Maoz, D., Nagar, N. M., Falcke, H. \& Wilson, A. S. 2005, ApJ, 625, 699 